\title{
Política de Crédito do BNDES e Sustentabilidade de Crescimento do Setor Primário ${ }^{1,2}$
}

\author{
Andrei Gomes Simonassi ${ }^{3}$, Ronaldo de Albuquerque e Arraes ${ }^{4}$ e \\ José Henrique Felix Silva ${ }^{5}$
}

Resumo: A proposta do artigo é investigar o efeito da oferta de crédito do Banco Nacional de Desenvolvimento Econômico e Social (BNDES) sobre a sustentabilidade de crescimento do setor primário, baseado em critérios de convergência e na aplicação de um modelo autorregressivo com valor limite endógeno, como proposto em Caner e Hansen (2001). Os exercícios empíricos foram conduzidos para o período de janeiro/2000 a setembro/2014 com dados trimestrais do Instituto Brasileiro de Geografia e Estatística (IBGE) para o Produto Interno Bruto (PIB) do setor primário e do BNDES para os desembolsos destinados a este setor. As estimativas permitem inferir que a dinâmica de crescimento da razão entre os desembolsos do BNDES e o PIB primário é linear e globalmente explosiva. Em conjunto, tais achados sugerem que a política de desembolsos do BNDES para o setor primário não é consistente com a manutenção de uma trajetória de crescimento econômico sustentável no longo prazo e, portanto, explicita indícios de ineficiência na concessão e alocação do crédito ofertado.

Palavras-chaves: Sustentabilidade de crescimento, setor primário, desembolsos BNDES, Valor Limite Endógeno.

Abstract: The purpose of the paper is to investigate the effectiveness of credit supply by the National Bank for Social and Economic Development (BNDES) on the growth sustainability of the primary sector, based on convergence criteria with the application of an autoregressive model with endogenous threshold, as proposed in Caner e Hansen (2001). The empirics were conducted in the period from January/2000 to September/2014 with quarterly data from the Brazilian Institute of Geography and Statistics (IBGE) for the Gross Domestics Product (GDP) of the primary sector and from the BNDES for disbursements to that sector. The estimates allow us to infer that the growth dynamics of

DOI - http://dx.doi.org/10.1590/1234-56781806-94790550102

1. Data de submissão: 14 de maio de 2015. Data de aceite: 29 de agosto de 2016.

2. Os autores agradecem ao CNPq.

3. Universidade Federal do Ceará (Caen/UFC) e pesquisador do CNPq. Fortaleza (CE), Brasil. E-mail: agsimonassi@ufc.br

4. Universidade Federal do Ceará (Caen/UFC) e pesquisador do CNPq. Fortaleza (CE), Brasil. E-mail: ronald@ufc.br

5. Universidade Federal do Ceará (UFC), Fortaleza (CE), Brasil. E-mail: hfelix@ufc.br 
the proposed indicator, disbursement/GDP, is linear and globally explosive. These findings suggest that BNDES disbursements policy for the primary sector is not consistent with maintaining a trajectory of sustainable economic growth in the long run, which leads to inefficiency in its allocation of credit granting.

Key-words: Growth sustainability, primary sector, BNDES credit granting, endogenous threshold.

Classificação JEL: C24, Q01, Q14, Q18.

\section{Introdução}

A literatura sobre a atuação dos bancos públicos para promoção do desenvolvimento econômico relata abordagens divergentes acerca da função e eficácia destas instituições para atingir esse objetivo. Por um lado, Lewis (1955), Myrdal (1960), Gerschenkron (1962) e Francisco et al. (2008) defendem o fornecimento de crédito para complementar as falhas deixadas pelo setor privado em economias com escassez de capital e com instituições fracas. Por outro lado, Schleifer e Vishny (1994) associam esta atuação como ferramentas utilizadas por políticos com objetivos de extrair ganhos de acordo com seus interesses, e a abordagem de De La Torre et al. (2006) preconizam que a alocação de créditos é direcionada para o cumprimento de políticas sociais.

No que diz respeito ao uso político dos bancos públicos, Francisco et al. (2008) admitem gerar malversação e má alocação de recursos, provocando a elevação dos custos decorrentes de burocracias governamentais e de práticas gerenciais ineficientes. Salomão (2010) afirma que as elites políticas e empresariais transgridem normas bancárias básicas, desrespeitam critérios prudenciais de operações, financiam o erário e emitem moeda escritural acima dos limites preestabelecidos.

Outra vertente da literatura sobre os bancos públicos aborda o comportamento inerentemente cíclico dos mercados, ao tratar os movimentos tanto de contração quanto de expansão, imprimindo uma lógica alternativa à dos agentes de mercado e reduzindo as desigualdades que são geradas e reforçadas pelos agentes de mercado, sejam elas setoriais ou regionais (SALOMÃO, 2010).

Um histórico recente da atuação dos bancos públicos pelo mundo pode ser dividido em quatro períodos distintos: o primeiro, nas décadas de 1950 e 1960, quando parte deles foi criada, inclusive no Brasil, seguia o ideário intervencionista Keynesiano que ainda predominava e cuja consequência foi um aumento na participação pública nos ativos totais dos maiores bancos, alcançando, na década de 1970, 50\% nos países desenvolvidos e 70\% nos países em desenvolvimento (YAYATI, MICCO e PANIZZA, 2007); o segundo, durante os anos 1980 e 1990, quando a crescente disseminação das concepções neoliberais resultou em um amplo processo de privatizações (JEANNEAU, 2007) que operou no sentido contrário, reduzindo referida participação para $25 \%$ e $50 \%$ nos países mais e menos desenvolvidos, respectivamente (YAYATI, MICCO e PANIZZA, 2007): o terceiro, de 1998 a 2007, quando este fenômeno de retração continuou em menor escala ou se estabilizou; o quarto, a partir da crise de 2008-2009, quando o debate sobre o papel destes bancos se revitalizou e gerou agendas que incluíam, por um lado, a 
estatização de instituições financeiras privadas de grande porte em países com sistemas financeiros sólidos como os Estados Unidos e o Reino Unido e, por outro lado, as intervenções para enfrentamento e minimização dos efeitos danosos desta crise (DEOS e MENDONÇA, 2010).

No Brasil, a atuação dos bancos públicos acompanhou de forma sincronizada esta cronologia, participando intensamente do financiamento de infraestrutura, do desenvolvimento das regiões, das privatizações e, em missão anticíclica, para o enfrentamento da crise de 2008-2009. Não obstante, há hiato na literatura que identifique a eficácia dessa atuação sobre os setores produtivos da economia, bem como se estes recursos despendidos pelo governo contribuem com a sustentabilidade de uma trajetória de crescimento. Tendo em vista os desajustes, má alocação e inadequados encaminhamentos que se tem constatado sobre a gestão dos recursos públicos em anos recentes, mormente severo ajuste fiscal que tem sido imposto pela administração central do País para equilibrar as contas públicas, essa lacuna motiva a realização do presente estudo, que agrega ainda ao subsidiar a avaliação acerca da eficiência na gestão dos recursos públicos no Brasil.

Ademais, cabe destacar que o intenso debate político-econômico recente pôs em cheque a atuação do Banco Nacional de Desenvolvimento Econômico e Social (BNDES) brasileiro enquanto instituição de fomento ao crescimento econômico do País.

Assim sendo, este artigo limita a investigação aos recursos administrados pelo BNDES e seus impactos na manutenção do crescimento econômico do setor primário da economia brasileira, considerando a hipótese de sustentabilidade do seu crescimento como resultante de uma convergência da evolução entre os desembolsos realizados à atividade e o PIB do respectivo setor no período de jan/2000 a set/2014.

A metodologia utilizada combina noções de cointegração e convergência a um modelo autorregressivo com threshold endógeno para estabelecer condições para sustentabilidade e captar os eventuais impactos dos desembolsos do
BNDES sobre o crescimento econômico do setor analisado.

Além desta introdução, o artigo apresenta cinco seções, contendo, em sequência, a fundamentação teórica na literatura, as evidências sobre as variáveis utilizadas e os indicadores desenvolvidos para investigar a hipótese de sustentabilidade do crescimento, a metodologia, discussão dos resultados e, por fim, as considerações finais.

\section{Abordagens sobre sustentabilidade}

De acordo com o Brundtland Report (1987), a definição mais geral de sustentabilidade leva em conta o aspecto intergeracional de responsabilidades no sentido que as escolhas de produção e consumo presentes dos agentes econômicos não devem comprometer as das gerações futuras. A literatura admite a existência de diferentes dimensões de um conceito para outro no caso da sustentabilidade agrícola, embora o pilar central seja a valorização dos recursos internos dos sistemas agrícolas produtivos (EDWARDS et al., 1990). Estas dimensões para avaliar sustentabilidade referem-se, segundo Sachs $(1990,2000)$, às esferas social, cultural, ecológica, ambiental e econômica. López-Ridaura et al. (2002) complementam afirmando que, para ocorrer sustentabilidade, ações são necessárias para implementar e consolidar princípios complexos e diversos e transformá-los em recomendações para a tomada de decisão dos agricultores.

Para Camino e Müller (1993), sustentabilidade na agricultura se define como a manutenção da produtividade de um agrossistema diante de choques exógenos de natureza econômica, climática ou outra ação perturbadora. Para tanto, elencam um conjunto de determinantes da sustentabilidade agrícola, tais como população, produção, consumo, emprego, tecnologia, produtividade, acesso e escoamento produtivo, rentabilidade, instituições e tempo. Já Rezende (1986, 1988), Carvalho (1989), Dias (1989), Guasquez e Villa Verde (1990), Goldin e Rezende (1990), Ferreira Filho (1994) seguem uma linha 
de pesquisa relacionando a sustentabilidade da agricultura com o ajustamento macroeconômico, choques externos, desempenho agregado, crescimento, movimento de preços agrícolas e oferta de alimentos.

O relatório anual 2008 do Banco Mundial dispõe que a agricultura pode ser um setor importante para o crescimento do Produto Interno Bruto (PIB) dos países em desenvolvimento e aponta como exemplo a África Subsaariana e, mais recentemente, China, Índia e Vietnã, cujo crescimento do setor agrícola permitiu impulsionar com sucesso o setor de transformação estrutural. Adianta ainda a necessidade de equilibrar a produção de alimentos, exportações tradicionais e de produtos com maior valor agregado, inclusive animais, como elementos favorecedores das bases para o crescimento sustentável do setor agrícola e, por consequência, dos demais setores da economia. Para tanto, sugere quatro elementos principais de política: equilíbrio ótimo entre impostos e incentivos; mais e melhores investimentos em agricultura; instituições mais fortes e eficazes e garantir o uso sustentável dos recursos naturais.

Para Gomes et al. (2009),

"a sustentabilidade dos sistemas agrícolas de produção traduziu-se, historicamente, pela manutenção da produtividade ao longo do tempo. Como evolução desse conceito, foram acrescidas ideias de estabilidade da produtividade e de equidade dos sistemas de produção (medida pela distribuição estatística de parâmetros como renda, número de propriedades e população). Na agricultura, a questão da sustentabilidade envolve fatores físicos, bióticos e aspectos relativos à viabilidade econômica e sociocultural" (p. 24).

Gomes et al. (2009) também afirmam que há diversas abordagens para avaliar a sustentabilidade agrícola, dentre as quais as que propõem a construção de índices agregados multidimensionais que avaliem o desempenho do sistema produtivo. Nesta abordagem, podem ser citados alguns trabalhos como Pannell e Glenn
(2000), Rigby et al. (2001), López-Ridaura et al. (2002), Lopes e Almeida (2003), Pacini et al. (2003), Fernandes (2004), Gomes (2004) e Parra-Lopez et al. (2008).

Fernandez-Sanchez et al. (2011) apresentam e comparam os principais marcos metodológicos de análise da sustentabilidade da agricultura mediante indicadores nacionais e internacionais, elucidando sua importância, tipologias e grau de aplicação, seguindo o trabalho de Kammerbauer (2001) e classificando os marcos metodológicos de avaliação da sustentabilidade em analíticos, sistêmicos e normativos e conclui que um mix destes poderia ser usado conforme o caso estudado.

A despeito de evidenciar a redução da participação da agricultura no PIB em passado recente, Sivakumar (2013) confirma a importância da Formação Bruta de Capital e do Produto Interno Bruto nas mudanças no setor agrícola da Índia e conclui que a agricultura necessita de políticas de perpetuação dos investimentos para garantir uma taxa de crescimento sustentável. Para a economia brasileira, Bonelli (2008) havia feito similar análise, enfatizando que a concretização do crescimento sustentado se daria a partir dos investimentos.

Mani et al. (2011) estimaram equações para os produtos dos setores agrícola, industrial e de serviços em termos dos investimentos público e privado e seus efeitos intersetoriais e na economia como um todo da Índia, no período de 1970 a 2008. Os resultados indicaram significativo impacto crowding-in do investimento público sobre o investimento privado em ambos os setores agrícola e industrial e, em diferentes cenários, mostraram substancial impacto crowding-in do investimento público sobre a agricultura.

Em oposição aos autores que acreditam que o crescimento sustentável de um setor produtivo resulta dos investimentos destinados ao mesmo, Heal (2011) afirma que não há como o PIB indicar sustentabilidade e sugere uma medida da evolução do total da riqueza de uma nação que considere todas as formas de capital: natural, físico, humano e social. Neste sentido, o autor usa uma alternativa criada por Hamilton 
e Hartwick (2005) e desenvolvida pelo Banco Mundial $(2006,2010)$ denominada Adjusted Net Savings-ANS para medir as mudanças no total de riqueza de um país. Conclui que, em termos econômicos, há um grau limitado de substituição entre o capital natural e as outras formas de capital, e que a ANS é apenas razoável como medida de sustentabilidade. Warner (2014), com dados do World Economic Outlook do Fundo Monetário Internacional (FMI), estudou os efeitos do investimento público sobre o crescimento econômico para uma amostra de 124 países com rendas baixas e médias. Concluiu que há pequenas e instantâneas associações positivas entre os booms dos investimentos públicos e crescimento econômico no curto prazo, e que estes ciclos de investimentos originados do governo não são sustentáveis no longo prazo.

Em relação ao fomento do crescimento e desenvolvimento, Pamplona (2011) destaca a continuidade da atuação do BNDES enquanto financiador do investimento no Brasil para a década de 2010, se constituindo no instrumento mais relevante para a execução da política do governo federal no apoio a programas, projetos, obras e serviços que se relacionem com o desenvolvimento econômico e social do nosso País. O fato é que o intenso debate político-econômico recente pôs em cheque o que é considerada a visão desta instituição e, em particular, o sentido de promover o desenvolvimento sustentável da economia brasileira.

$\mathrm{Na}$ linha dos trabalhos empíricos que investigam os efeitos dos desembolsos de instituições financeiras públicas, Wegelin (2014) utiliza três indicadores - PIB, PIB per capita e o Índice Firjan de emprego e renda - para medir tais efeitos, tendo por base os municípios brasileiros no período 2000-2011. Conclui que nos municípios em que houve aumento do crescimento médio anual do desembolso do BNDES no período 2006-2011, comparado a 2000-2005, o PIB e o PIB per capita variaram positivamente, enquanto o índice Firjan tenha se mostrado indefinido. Para esses municípios, o PIB e o PIB per capita cresceram, em média, $0,4 \%$ ao ano a mais do que os demais, e benefício gerado para cada $\mathrm{R} \$ 1,00$ de desembolso do
BNDES foi, em média, de R \$ 0,29 de acréscimo no PIB. Comparando-se os benefícios gerados pelo BNDES na economia e o custo de oportunidade para o Tesouro Nacional em optar por financiar a operação do BNDES, a autora demonstra que o retorno total líquido sobre $\mathrm{R} \$ 1,00$ que o Tesouro toma emprestado para repassar ao BNDES é, em média, $-75,84 \%$.

A proposta apresentada nesta pesquisa é correlata à de Wegelin (2014), porém, com a especificidade de focar um setor econômico. $\mathrm{O}$ indicador definido como a razão entre os desembolsos do BNDES e o PIB do setor primário dará suporte à investigação da evolução e a sustentabilidade do crescimento da atividade agropecuária, sustentabilidade esta definida sob a ótica de manutenção ou continuidade de uma trajetória de crescimento econômico do setor.

\section{Evidências e um indicador para mensurar sustentabilidade}

A literatura define políticas anticíclicas como aquelas que revertem situações recessivas, de crises ou depressões, enquanto as cíclicas visam a manutenção de cenários de crescimento econômico desejáveis em prazos mais longos em termos do produto, renda e emprego. Especialmente em países em desenvolvimento como o Brasil, os bancos públicos assumem um papel fundamental na execução dessas políticas, cuja intervenção tem como finalidade suavizar os cíclicos da atividade econômica.

Salomão (2010) comenta os efeitos da crise mundial de 2008 na economia brasileira e considera como decisiva a atuação dos bancos públicos em 2009 para reverter o iminente cenário de recessão. Mesmo economias que ainda apresentaram crescimento significativo em 2009, como a China e a Índia, também tiveram uma política anticíclica ativa por parte dos bancos públicos.

Para evidenciar a atuação dos bancos públicos, particularmente do BNDES, cujos desembolsos para o setor primário são utilizados neste estudo, a Tabela 1 e a Figura 1 apresentam, 
Tabela 1. Desembolsos do BNDES por setor de atividade em 2014

\begin{tabular}{lcc}
\hline \multicolumn{1}{c}{ Setor } & \% do total de desembolsos & Valor em R\$ bilhões \\
\hline 1o Transportes terrestres $^{\text {o }}$ Eletricidade e gás & 15,0 & 28,1 \\
3o Comércio & 10,4 & 19,6 \\
$4^{\text {o }}$ Agropecuária & 9,4 & 17,6 \\
\hline
\end{tabular}

Fonte: BNDES.

Figura 1. Desembolsos do BNDES ao Setor Primário (2000: T1 a 2014:T3)

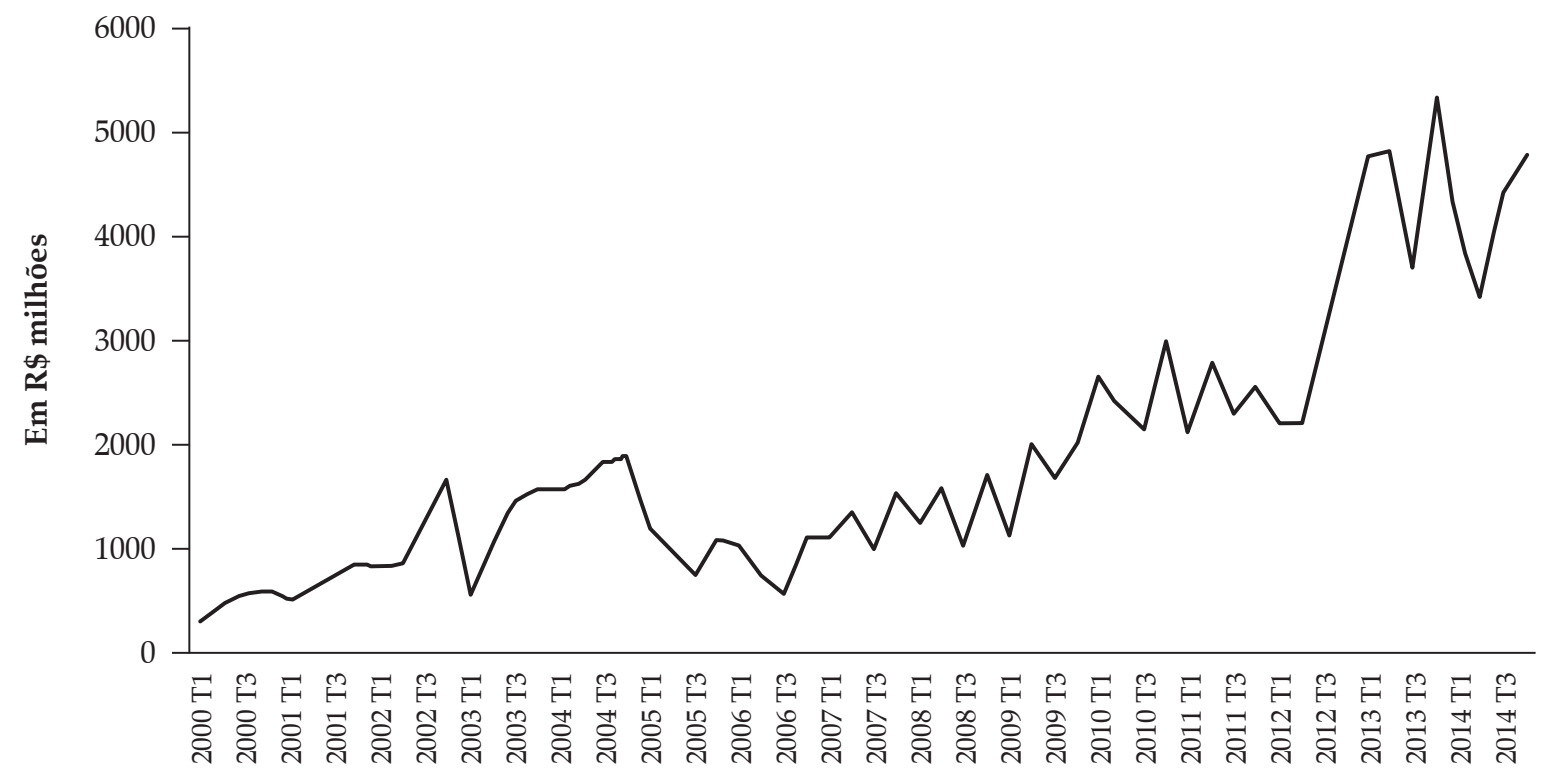

Fonte: BNDES.

respectivamente, os principais setores em alocação de recursos por parte do BNDES com base no ano de 2014 e a evolução trimestral dos desembolsos ao setor primário entre o primeiro trimestre de 2000 e o terceiro trimestre de 2014.

Observa-se que a atividade agropecuária representa $8,9 \%$ do total de recursos disponibilizados pelo BNDES e que, de acordo com o valor de 16,8 bilhões de reais desembolsado pela instituição em 2014, se mostra como prioritária, estando atrás apenas de setores chave em infraestrutura, como Transporte e Energia, e do setor Comércio. As informações disponibilizadas no sítio do BNDES demonstram ainda que $75,2 \%$ dos recursos destinados ao setor primário são para micro e pequenas e $15,0 \%$, para grandes empresas.
Além disso, constata-se na Figura 1 a elevação do montante de recursos destinados à Agropecuária, principalmente a partir de 2009, ano subsequente à crise financeira internacional. Anualizando as informações do referido gráfico, tem-se desembolsos que possuem valores nominais de $\mathrm{R} \$ 5,6$ bilhões em 2009 e atingem os R $\$ 16,8$ bilhões em 2014, fato que explicita que o estímulo ao setor deve compreender uma das diretrizes da política econômica do governo.

Para maior sedimentação da análise e suporte metodológico cabe inspecionar a evolução dos desembolsos vis à vis, o PIB do setor em que tais recursos são alocados, a fim de avaliar os possíveis impactos sobre o crescimento econômico. Para tanto, conforme descrito nas seções anteriores, este estudo analisa a dinâmica de um 
indicador definido pela razão desembolsos/PIB do setor primário do Brasil, no período de 2000 a 2014, seguindo o modelo autorregressivo com valor limite endógeno (Threshold Autoregressive Model) introduzido por Caner e Hansen (2001).

A Figura 2 ilustra a trajetória do indicador em termos percentuais, que será a variável de interesse utilizada no exercício empírico da seção seguinte. Cabe destacar que é notória a mudança de tendência do referido indicador após 2009, a partir de quando se verifica um crescimento exponencial dos desembolsos, uma vez que o PIB nacional demonstrava sinais de arrefecimento, conforme explicitado na Figura 1. Estes fatos estilizados motivam e justificam, neste estudo, a escolha de uma técnica apropriada para identificar mudanças de regimes dos indicadores analisados.

\section{Metodologia}

\subsection{Base de dados}

Foram utilizados dados trimestrais do IBGE para o produto do setor primário e os desem- bolsos do BNDES destinados a este setor, abrangendo o período jan/2000 - set/2014, convertidos em frequência trimestral. A escolha deste período se justifica pela necessidade de analisar dois períodos recentes e distintos do ponto de vista do financiamento público das atividades do setor primário brasileiro, tendo em conta sua retomada gradual após as crises que dominaram os cenários externo e interno das economias em desenvolvimento no final da década de 1990 e, de forma mais explosiva, a partir da crise de 2008 das economias desenvolvidas.

O indicador utilizado é definido pela razão desembolsos/PIB do setor. O exercício empírico segue o modelo autorregressivo com valor limite endógeno proposto por Caner e Hansen (2001).

\subsection{O modelo econométrico}

O procedimento metodológico proposto para avaliar a sustentabilidade do crescimento do setor primário brasileiro se baseia na aplicação de um modelo autorregressivo com valor limite endógeno (Threshold Autoregressive Model) introduzido

Figura 2. Evolução dos desembolsos como proporção do PIB do Setor Primário (2000:T1 - 2014:T3)

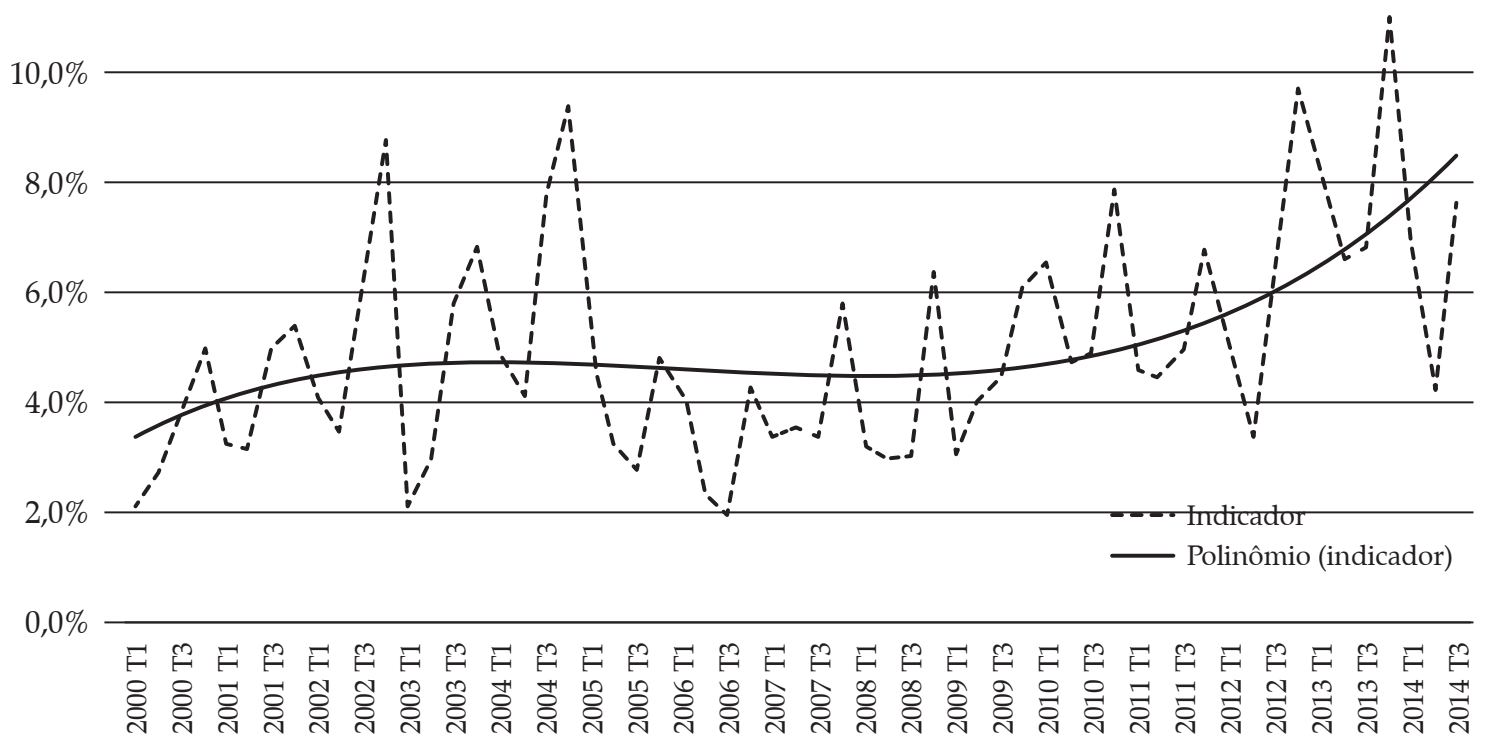

Fonte: Dados do IBGE e BNDES, estimativas próprias. 
por Caner e Hansen (2001) ${ }^{6}$, com a seguinte especificação econométrica:

$\Delta y_{t}=\theta_{1}{ }^{\prime} x_{t-1} I_{\left(\mathrm{Z}_{t-1}<\lambda\right)}+\theta_{2}{ }^{\prime} x_{t-1} I_{\left(\mathrm{Z}_{t-1} \geq \lambda\right)}+\varepsilon_{t} \quad t=1, \ldots T$

em que $x_{t-1}=\left(y_{t-1}, r_{t}^{\prime}, \Delta y_{t-1}, \ldots, \Delta y_{t-k}\right)^{\prime}, I_{(.)}$é uma função indicador com $I_{(x)}=1$ se $x \in A$; o erro $\varepsilon_{t}$ segue a usual hipótese i.i.d.; $Z_{t}=y_{t}-y_{t-m}$ para $m \geq 1$; $r_{t}$ é um vetor de componentes determinísticos incluindo a constante e possivelmente uma tendência linear.

$\mathrm{O}$ parâmetro $\lambda$ representa o valor limite (threshold). Embora desconhecido, assume valores no intervalo $\Lambda=\left[\lambda_{1}, \lambda_{2}\right]$, em que $\lambda_{1}$ e $\lambda_{2}$ são escolhidos de forma que $\operatorname{Prob}\left(Z_{t} \leq \lambda_{1}\right)=\pi_{1}$ e $\operatorname{Prob}\left(Z_{t} \leq \lambda_{2}\right)=\pi_{2}$, desde que $\pi_{1}$ e $\pi_{2}$ sejam complementares, ou seja, $\pi_{1}=1-\pi_{2}$ Impõe-se, com essa restrição, que nenhum regime terá um tamanho de subamostra inferior à proporção $\pi_{1}$ do total da amostra. Caner e Hansen (2001) sugerem que $\pi_{1}=0,15$, o que implica que nenhum dos regimes terá menos que $15 \%$ das observações ${ }^{7}$.

Por fim, define-se $\theta_{1}=\left(\rho_{1}, \beta_{1}, \alpha_{1}\right)^{\prime}$ e $\theta_{2}=\left(\rho_{2}\right.$, $\left.\beta_{2}, \alpha_{2}\right)^{\prime}$, em que $\rho_{1}$ e $\rho_{2}$ são escalares, $\beta_{1}$ e $\beta_{2}$ têm a mesma dimensão de $r_{t}$ e $\alpha_{1}$ e $\alpha_{2}$ são vetores de dimensão $k$. Portanto, $\rho_{1}$ e $\rho_{2}$ são coeficientes de $y_{t-1}, \beta_{1}$ e $\beta_{2}$ são coeficientes dos componentes determinísticos, e $\alpha_{1}$ e $\alpha_{2}$ são os coeficientes de $\left(\Delta y_{t-1}, \ldots, \Delta y_{t-k}\right)$ nos regimes 1 e 2 , respectivamente.

A opção por restringir a classificação das observações em dois regimes atende ao interesse de se investigar uma mudança de padrão na atuação do BNDES na destinação dos seus desembolsos para a atividade agropecuária, em especial em períodos de instabilidade econômica. Obviamente, a opção por múltiplos regimes não

6. No modelo original, os autores utilizam a ferramenta para analisar a dinâmica da taxa de desemprego norte-americana.

7. A amostra utilizada no estudo possui 58 observações e nenhum regime tem menos que 14 observações. Após as defasagens, o modelo foi estimado no regime $2 \mathrm{com}$ um mínimo de 17 observações para o setor agrícola (ver seção 5). apenas parece desnecessária ao propósito do estudo como compromete a obtenção de estimativas consistentes diante do limitado tamanho da amostra utilizada. Ademais, a metodologia escolhida permite ainda a possibilidade de testar simultaneamente a linearidade e a estacionaridade do indicador proposto.

Para implementar o método dos Mínimos Quadrados (MQ) na estimação da equação 1, Caner e Hansen (2001) sugerem aplicar o algoritmo da concentração, que consiste em executar as seguintes operações:

a) Para cada $\lambda \in \Lambda$, a equação 1 é estimada por $\mathrm{MQ}$, ou seja, para cada $\lambda \in \Lambda$ tem-se:

$$
\Delta y_{t}=\hat{\theta}_{1}(\lambda)^{\prime} x_{t-1} I_{\left(Z_{t-1}<\lambda\right)}+\hat{\theta}_{2}(\lambda)^{\prime} x_{t-1} I_{\left(Z_{t-12 \lambda}\right)}+\hat{\varepsilon}_{t}(\lambda)
$$

com

$$
\hat{\sigma}^{2}(\lambda)=T^{-1} \sum_{t=1}^{T} \hat{\varepsilon}_{t}(\lambda)^{2}
$$

b) Estimar o valor limite, $\lambda$, minimizando $\sigma^{2}(\lambda)$, ou seja;

$\hat{\lambda}=\arg \min _{\lambda \in \Lambda} \hat{\sigma}^{2}(\lambda)$

c) Estimar $\theta_{1}$ e $\theta_{2}$ utilizando a estimativa de $\lambda$ em (b), $\hat{\lambda}$, isto é:

$\Delta y_{t}=\hat{\theta}_{1}(\hat{\lambda})^{\prime} x_{t-1} I_{\left(Z_{t-1}<\lambda\right)}+\hat{\theta}_{2}(\hat{\lambda})^{\prime} x_{t-1} I_{\left(Z_{t-1} \geq \lambda\right)}+\hat{\varepsilon}_{t}(\hat{\lambda})$

com

$$
\hat{\sigma}^{2}(\hat{\lambda})=T^{-1} \sum_{t=1}^{T} \hat{\varepsilon}_{t}(\hat{\lambda})^{2}
$$

As estimativas das equações 3.1 e 3.2 são de suma importância, pois serão utilizadas para conduzir inferência sobre os parâmetros da equação 1 através de estatísticas de teste do tipo " $t$ " e de Wald descritas a seguir. 


\subsubsection{A Análise da Dinâmica do Indicador Desembolsos/PIB}

Investigar a mudança de dinâmica no crescimento do indicador desembolsos/PIB para o setor estudado implica em testar, na equação 1 , se ocorre o seguinte:

$$
\hat{\theta}_{1}(\hat{\lambda})=\left(\hat{\rho}_{1}, \hat{\beta}_{1}, \hat{\alpha}_{1}\right)^{\prime} \neq \hat{\theta}_{2}(\hat{\lambda})=\left(\hat{\rho}_{2}, \hat{\beta}_{2}, \hat{\alpha}_{2}\right)^{\prime}
$$

Cabe notar que, na presença de linearidade, a regressão (3.1) se identifica como a usual regressão ADF (Augmented Dickey-Fuller).

Apesar de uma crítica às demais técnicas de investigação não ser o principal propósito do estudo, vale ressaltar que se a evolução do indicador desembolsos/PIB de cada setor possuir dinâmica não linear, testar a estacionaridade do processo descrito pela mesma via do teste tradicional de raiz unitária $\mathrm{ADF}$ pode culminar em resultados imprecisos. Por este motivo, um teste consistente para investigar esta evolução foi proposto por Caner e Hansen (2001), cuja estatística é dada por:

$$
W_{T}=W_{T}(\hat{\lambda})=\sup _{\lambda \in \Lambda} W_{T}(\lambda)
$$

em que $W_{T}(\lambda)=T\left(\frac{\sigma_{0}^{2}}{\hat{\sigma}^{2}(\hat{\lambda})}-1\right)$ e $\sigma_{0}^{2}$ representa a variância do resíduo obtido quando se estima a equação (1) impondo $H_{0}: \theta_{1}=\theta_{2}$, com $\hat{\sigma}^{2}(\hat{\lambda})$ calculado de acordo com (3.2).

A distribuição assintótica de $W_{T}$ sob estacionaridade, $\rho<0$, foi investigada por Davies (1987), Chan (1991), Andrews e Ploberger (1994) e Hansen (1996). Caner e Hansen (2001) mostram que, sob a presença de raiz unitária, a distribuição assintótica de $W_{T}$ depende da estrutura dos dados, significando que os valores críticos não podem ser tabulados. Desta forma, os autores sugerem dois métodos de bootstrap para aproximar a distribuição assintótica de $W_{T}$ : o primeiro é apropriado para o caso estacionário, $\rho<0$, enquanto o segundo é mais adequado para o caso com raiz unitária, $\rho<0$. Dado que a ordem de integração é desconhecida a priori, os valores críticos são calculados por bootstrap assumindo $\rho=0$ e $\rho<0$, e seu valor mais conservador, maior p-valor, é aplicado na estimação. Portanto, este será o procedimento utilizado neste artigo para testar a hipótese nula de linearidade, qual seja, $H_{0}: \theta_{1}=\theta_{2}$.

\subsubsection{Hipótese de raiz unitária em modelos não lineares}

Os parâmetros $\rho_{1}$ e $\rho_{2}$ da equação 1 controlam a estacionaridade do processo $y_{t}$, o qual será integrado de ordem $1, I(1)$, se a hipótese nula, $H_{0}$ $: \rho_{1}=\rho_{2}=0$, for verdadeira. Uma hipótese alternativa natural é dada por, $H_{1}: \rho_{1}<0$ e $\rho_{2}<0$, pois denotaria o processo $\left(y_{t}\right)$ estacionário nos dois regimes. Outra possibilidade mais flexível para formulação da hipótese alternativa e deveras útil para fins de política, denomina-se raiz unitária parcial, cuja especificação é dada por:

$$
H_{2}:\left\{\begin{array}{c}
\rho_{1}<0 \text { e } \rho_{2}=0 \\
\text { ou } \\
\rho_{1}=0 \text { e } \rho_{2}<0
\end{array}\right.
$$

se $H_{2}$ for verdadeira, o processo $y_{t}$ terá raiz unitária em um dos regimes, mas será estacionário no outro. Três testes ${ }^{8}$ para investigar a estacionaridade do crescimento do indicador desembolsos/ PIB do setor primário são, então, realizados: raiz unitária global e dois outros para captar a presença de raiz unitária em cada regime, de acordo com as estatísticas $R_{1 T}, t_{1}$ e $t_{2}$.

Tal discriminação é extremamente importante, pois permite examinar se o crescimento do indicador desembolsos/PIB utilizado apresenta mudança de regime, ou seja, de estacionário para não estacionário ou vice-versa, após atingir certo valor limite, $\lambda$, abaixo do qual estão as observações do regime 1 e, acima deste, as observações do regime 2. Associada à investigação acerca da possibilidade de mudança de regime deve estar a análise da tendência de longo prazo de cada indicador, com as seguintes possibilidades:

8. Ver detalhes no apêndice. 
- Caso 1: A estacionaridade do indicador sugere que a variação dos desembolsos segue a variação do PIB de cada setor e, portanto, existe cointegração entre estas componentes, de forma que a política de desembolsos do BNDES é condizente com uma trajetória sustentável de crescimento de longo prazo;

- Caso 2: Não estacionaridade com tendência positiva sugere que a evolução dos desembolsos é relativamente superior à evolução do PIB, de forma que, mesmo no caso da manutenção de um montante elevado de desembolsos como mecanismo anticíclico, esta política do BNDES não está sendo eficiente no estímulo ao crescimento econômico setorial;

- Caso 3: Não estacionaridade com tendência negativa sugere que a evolução dos desembolsos é relativamente inferior à evolução do PIB. Neste caso, o crescimento econômico pode estar gerando os recursos necessários para investimentos ou há um aumento menos que proporcional nos desembolsos, quando comparado ao produto setorial. Em ambos os casos, a política adotada pelo BNDES não deve ser compatível com uma trajetória de crescimento sustentável no longo prazo.

\section{Resultados}

A Tabela 2 sumariza os resultados, na qual estão dispostos os testes de linearidade e de igualdade dos coeficientes, as estimativas do modelo irrestrito $^{9}$ nos dois regimes ${ }^{10}$, o limiar para os regimes $(\lambda)$, o número de observações $(N)$, o número de defasagens ótimo ${ }^{11}(m)$ para a variável

9. O modelo restrito assume a presença de raiz unitária na equação 1.

10. Regime 1 consiste em $Z_{t-1}<\hat{\lambda}$, enquanto o regime 2 consiste em $Z_{t-1} \geq \hat{\lambda}$

11. A escolha de " $m$ " é realizada a partir do valor que maximiza $W_{T}$. Mais detalhes sobre estimação de " $m$ " estão em Caner e Hansen (2001).
$Z_{\mathrm{t}}=y_{\mathrm{t}}-y_{\mathrm{t}-\mathrm{m}}{ }^{12}$, o número ótimo de defasagens $(k)$, na regressão $\mathrm{ADF}^{13}$ (equação 1), e os valores críticos ${ }^{14}$ para os testes de linearidade. Todo o processo de estimação e computação de testes de hipóteses foi implementado através de programação em GAUSS.

Observa-se, nessa tabela, que a dinâmica da taxa de crescimento do indicador desembolsos/ PIB do setor primário é linear, indicando que não há mudança de política por parte do BNDES, e o número ótimo de defasagens é $m=1$ com valor limite $\hat{\lambda}=0,0123$. Isso significa que, no termo $Z_{t}=y_{t}-y_{t-1}$ da equação 1 , variações inferiores a 1,23 p.p. no crescimento trimestral do indicador desembolsos/PIB do setor primário caracterizam as observações do regime 1 , caso contrário, as observações do regime 2 . Não obstante as estimativas da equação ADF em cada regime e, dada a diferenciação constatada através do teste de Wald de linearidade, perde-se importância a comparação das estimativas nos dois regimes, mesmo quando estatisticamente significantes. Ressalta-se que, de acordo com os testes de Wald para igualdade dos coeficientes, há divergência entre os coeficientes da defasagem e da segunda diferença nos dois regimes.

Uma vez não rejeitada a hipótese nula de linearidade, investigou-se a presença de raiz unitária nas séries dos indicadores. Foram calculadas as estatísticas $R_{1 T}, t_{1}$ e $t_{2}$ para $m=1$, e reportados tanto os p-valores assintóticos como aqueles derivados pelo método bootstrap. Mesmo com uma amostra de 56 observações após as defasagens utilizadas, a opção por uma frequência trimestral faz com que os p-valores obtidos pelo método

12. É importante observar que a variável limiar não precisa ser apenas o crescimento do indicador desembolsos/PIB. Poderia ser utilizado apenas o indicador, mas acredita-se que o nível considerado desejável pode variar de acordo com o momento econômico, já a variação do indicador em cada momento é que deve ser a métrica do BNDES para sua política de desembolsos e, por isso, esta foi a variável escolhida na análise. Outro ponto é que a não rejeição da hipótese de linearidade pode ser causada pelo uso de uma variável limiar que não seja estritamente estacionária sob Ho.

13. Calculado de acordo com o critério GS (do geral para o específico - "general to specific").

14. Ver valor em + na nota abaixo da tabela. 
Tabela 2. Estimativas do modelo irrestrito e teste de linearidade

\begin{tabular}{|c|c|c|c|c|c|c|}
\hline & \multicolumn{4}{|c|}{ Estimativas, $\hat{m}=1, \hat{\lambda}=0,0123$} & \multicolumn{2}{|c|}{ Teste para igualdade dos coeficientes } \\
\hline & \multicolumn{2}{|c|}{$Z_{t-1}<\hat{\lambda}$} & \multicolumn{2}{|c|}{$Z_{t-1} \geq \hat{\lambda}$} & \multirow{2}{*}{$\begin{array}{c}\text { Estatística } \\
\text { de Wald }\end{array}$} & \multirow{2}{*}{$\begin{array}{l}\text { Bootstrap } \\
\text { P-valor }\end{array}$} \\
\hline & Estimativa & $\sigma$ & Estimativa & $\sigma$ & & \\
\hline Intercepto & 0,0202 & 0,0105 & $0,0207^{* *}$ & 0,0184 & 0,0006 & 1,0000 \\
\hline$y_{t-1}$ & $-0,3020^{* *}$ & 0,2180 & $-0,2320^{* *}$ & 0,4350 & 0,0207 & 0,0900 \\
\hline$y_{t-1}$ & $0,0817^{* *}$ & 0,1940 & $-0,4720^{* *}$ & 0,8540 & 0,4000 & 0,5000 \\
\hline$\Delta y_{t-2}$ & $-0,3260$ & 0,1380 & $-1,1300$ & 0,5000 & 2,4300 & 0,0000 \\
\hline$N$ & \multicolumn{2}{|c|}{39} & \multicolumn{2}{|c|}{17} & & \\
\hline \multicolumn{5}{|c|}{ Teste conjunto de linearidade (Wald para Valor Limite) } & $5,0300^{+}$ & 0,9600 \\
\hline \multicolumn{5}{|c|}{$\mathrm{N}$} & \multicolumn{2}{|c|}{56} \\
\hline
\end{tabular}

Notas: ${ }^{* *}$ Não significante a $5 \%$; + Valor Crítico a $5 \%=16,2$.

Fonte: Estimativas próprias.

bootstrap possam ser particularmente importantes para garantir a robustez dos resultados, conforme expostos na Tabela 3.

Tabela 3. Testes de raiz unitária para os dois regimes

\begin{tabular}{cccc}
\hline \multirow{2}{*}{ Testes } & \multirow{2}{*}{ Estatística } & \multicolumn{2}{c}{ p-valor } \\
\cline { 3 - 4 } & & Assintótico & Bootstrap \\
\hline$R_{1 T}$ & 2,2100 & 0,8900 & 0,7000 \\
$t_{1}$ & 1,3900 & 0,7490 & 0,3900 \\
$t_{2}$ & 0,5340 & 0,9400 & 0,6000 \\
\hline
\end{tabular}

Fonte: Estimativas próprias.

O resultado da estatística $R_{1 T}$, não significante a $5 \%$, indica que o crescimento da razão desembolsos/PIB primário é globalmente explosivo, confirmação atestada tanto pelos valores críticos assintóticos quanto por bootstrap. Os baixos valores das estatísticas $t_{1}$ e $t_{2}$ implicam que, mesmo se a dinâmica fosse não linear, não haveria estacionaridade nos dois regimes. Sob as hipóteses estabelecidas anteriormente, tais resultados sugerem uma inconsistência da política de desembolsos do BNDES com uma trajetória de crescimento sustentável para o setor.

Em conjunto, os resultados demonstram que a hipótese nula da existência de raiz unitária não é rejeitada, implicando que, independente dos regimes, o indicador desembolsos/PIB segue um processo explosivo. Portanto, pode-se inferir que a política de desembolsos do BNDES para atividade agropecuária não é consistente com a manutenção de uma trajetória de crescimento econômico sustentável no longo prazo.

\section{Considerações finais}

O papel dos bancos públicos na promoção do desenvolvimento econômico dos países é assunto de persistente debate na literatura. Especialmente em momentos de pouca liquidez ou elevada incerteza, o setor público precisa compensar a escassez de recursos para investimentos. A literatura sobre este tema enfatiza ainda a importância da Formação Bruta de Capital no crescimento de uma economia e a necessidade das autoridades governamentais, por meio dos bancos públicos, perpetuarem políticas de investimentos para garantir uma taxa de crescimento sustentável.

Visando contribuir com o debate, este estudo analisou a dinâmica de crescimento de um indicador definido pela razão desembolsos do BNDES/ PIB para o setor primário brasileiro considerando-se a hipótese de sustentabilidade do crescimento deste setor no Brasil como resultante de uma convergência da evolução entre os desembolsos realizados à atividade e o PIB do respectivo setor. O conceito de sustentabilidade proposto se dá, então, sob a ótica de manutenção ou continuidade deste crescimento econômico.

Para tanto, foram utilizados dados trimestrais do IBGE para o PIB do setor primário e do próprio BNDES para os desembolsos destinados a 
este setor, abrangendo o período de janeiro/2000 a setembro/2014.

Os dados amostrais revelaram que a trajetória para a variação percentual do indicador utilizado apresenta movimentos com evidente reversão à média a partir do segundo trimestre de 2008. Estes fatos motivaram e fundamentaram a aplicação de um modelo autorregressivo com valor limite endógeno capaz de identificar eventuais mudanças de regimes dos indicadores analisados.

Os resultados permitiram observar que a dinâmica da taxa de crescimento do indicador desembolsos/PIB do setor primário é linear e segue um processo globalmente explosivo. Tais constatações coadunam com os achados de Wegelin (2014), ao atestar que a política de desembolsos do BNDES no Brasil é ineficiente para estimular e manter uma trajetória de crescimento econômico sustentável para geração de emprego e renda em nível municipal no longo prazo, e com os de Warner (2014), quando afirma que os ciclos de investimentos originados do governo não são sustentáveis no longo prazo.

A hipótese subjacente ao exercício empírico realizado reside no fato de que, se o indicador segue um processo estacionário, há uma trajetória de crescimento sustentável no longo prazo para o setor, pois há indícios de convergência entre os desembolsos realizados e o PIB do setor. De outra forma, uma trajetória explosiva implica em comprometimento da capacidade de crescimento, haja vista que a política do governo em termos de desembolsos do BNDES é incapaz de estimular o crescimento do setor. Estabelece-se, desta forma, um diagnóstico sobre os efeitos da gestão de recursos públicos para o setor produtivo da agropecuária brasileira, sugerindo, com isso, que a política de crédito para o setor deveria ser reavaliada para que pudesse vir a apresentar sustentabilidade no longo prazo.

Sugere-se pesquisas adicionais utilizando a mesma metodologia deste artigo para investigar a dinâmica de crescimento de outros setores econômicos da economia brasileira, bem como para permitir comparações entre países em termos da importância dos investimentos dos bancos públicos na sustentabilidade do crescimento destes setores.

\section{Referências}

ANDREWS, D. W. K. e PLOBERGER, W. Optimal Tests When a Nuisance Parameter Is Present Only under the Alternative. Econometrica, v. 62, p. 1383-1414, 1994.

BONELLI, R. Investimento e crescimento sustentado. IBRE/FGV, Rio de Janeiro, 2008.

CAMINO, V. R. e MULLER, S. Sostenibilidad de la agricultura y los recursos naturales. Bases para estabelecer indicadores. Série Documentos de Programas, N. 38. Instituto Interamericano de Cooperação para a Agricultura (IICA) e Ministério Federal Alemão de Cooperação Técnica (GTZ), San José (Costa Rica), 1993.

CANER, M. e HANSEN, B. E. Threshold Autoregression with a Unit Root. Econometrica, v. 69, p. 1555-1596, 2001.

CARPENTER, R. A. Can sustainability be measured? Environmental Strategy, v. 5, p. 13-16, 1993.

CARVALHO, J. L. Choques externos e resposta interna: semeando ventos e colhendo tempestades na agricultura brasileira. Revista Brasileira de Economia, v. 43, n. 2, p. 139-75, 1989.

CHAN, K. S. Percentage Points of Likelihood Ratio Tests for Threshold Autoregression. Journal of the Royal Statistical Society, series B, v. 53, p. 691-696, 1991.

DAVIES, R. B. Hypothesis Testing When a Nuisance Parameter is Present Only under the Alternative. Biometrika, v. 74, p.33-43, 1987.

DE LA TORRE, A., GOZZI, J. C., SCHMUKLER, S. L. Innovative Experiences in access to finance: market friendly roles for the invisible hand? Latin America Regional Studies Series, Concept Paper, World Bank, Washington, DC, 2006.

DEOS, S. e MENDONÇA, A. R. R. Uma proposta de delimitação conceitual de bancos públicos. Bancos Públicos e Desenvolvimento, cap. 2. IPEA, Rio de Janeiro, 2010.

DIAS, G. L. S. O papel da agricultura no processo de ajustamento: nota adicional. Anais do XXVII Congresso Brasileiro de Economia e sociologia Rural. Piracicaba, v. II, p. 310-317, 1989.

EDWARDS, C.A. et al. Sustainable agricultural systems. Iowa: Soil and Water Conservation Society, 1990. 
FERNANDES, L. A. O. The meaning of sustainability: searching for agri-environmental indicators. Thesis (Ph.D.), University of Manchester, 2004, 332p.

FERNANDEZ-SANCHEZ G. e MATOS, M. M. Marcos Metodológicos para sistematização de indicadores de sustentabilidade da agricultura. [SYN]THESIS, v. 5, n. 2, p. 255-267, 2012.

.et al. Análise da sustentabilidade da agricultura: proposta metodológica de indicadores para a agricultura fluminense. Belo Horizonte: $49^{\circ}$ Congresso SOBER, 2011.

FERREIRA FILHO, J. B. S. Notas a respeito do desempenho agregado da agricultura brasileira no período 1980-1991. Revista de Economia e Sociologia Rural, v. 32, n. 3, 1994.

FRANCISCO, M. et al. Measuring the performance and achievement of social objectives of development finance institutions. Policy Research Working Paper, n. 4506, World Bank, 2008.

GERSCHENKRON, A. Economic backwardness in historical perspective. Cambridge: Belknap Press of Harvard University Press, 1962.

GOLDIN, I. e REZENDE, G. C. Agriculture and economic crisis: lessons from Brazil. Paris: OCDE. Development Centre Studies, 1990.

GOMES, E. G. et al. Estudo da sustentabilidade agrícola em município amazônico com análise envoltória de dados. Pesquisa Operacional, v. 29, n. 1, Rio de Janeiro, jan./abr. 2009.

GOMES, I. Sustentabilidade social e ambiental na agricultura familiar. Revista de Biologia e Ciências da Terra, v. 5, n. 1, p. 1-17, 2005.

GUASQUEZ, J. G. e VILLA VERDE, C. M. Crescimento da agricultura brasileira e política agrícola os anos oitenta. Texto para Discussão, n. 204. Brasília: IPEA, 1990.

HAMILTON, K. e HARTWICK, J. M. Investing exhaustible resource rents and the path of consumption. Canadian Journal of Economics, v. 38, n. 2, p. 615-621, 2005.

HANSEN, B. E. Inference When a Nuisance Parameter is not Identified under the Null Hypothesis. Econometrica, v. 64, p. 413-430, 1996.

HEAL, G. Sustainability and its Measurement. NBER Working Paper, n. 17008, 2011.

JAYME JR. F. G. e CROCCO, M. Bancos Públicos e Desenvolvimento Econômico. Rio de Janeiro: IPEA, 2010.
KAMMERBAUER, J. Las dimensiones de la sostenibilidad: fundamentos ecológicos, modelos paradigmáticos y senderos. Interciencia, v. 26, n. 8 , p. 353-359, 2001.

KÖRNER, T. e SCHNEIBEL, I. Public ownership of banks and economic growth: the role of heterogeneity. CEPR Discussion Paper, n. 8138, London, Center for Economic Policy Research, 2010.

LA PORTA, R., LOPEZ-DE-SILANES, F. e SHLEIFER, Andrei. Government ownership of banks. Journal of Finance, v. 57, n. 1, p. 265-301, 2002.

LAZZARINI, S. G. et al. What do development banks do? Evidence from Brazil, 2002-2009, 2012. Disponível em: $<$ http://inctpped.ie.ufrj.br/spiderweb/dymsk_4/4-4 Lazzarini-What do DB do.pdf>. Acesso em: fev. 2015.

LEWIS, W. A. The theory of economic growth. Irwin: Homewood, Ill, 1955.

LOPES, S. B. e ALMEIDA, J. Methodology for comparative analysis of sustainability in agroforestry systems. Revista de Economia e Sociologia Rural, v. 41, n. 1, p. 183-208, 2003.

LÓPEZ-RIDAURA, S. et al. Evaluating the sustainability of complex socio-environmental systems: the MESMIS framework. Ecological Indicators, v. 2, p. 135-148, 2002.

LUNDBERG, E. L. Bancos oficiais e crédito direcionado: o que diferencia o mercado de crédito brasileiro? Banco Central do Brasil - Trabalhos para Discussão, n. 258, Brasília, 2011.

MANI, H. et al. Public investment in agricultural and GDP growth: another look at the intersectoral linkages and policy implications. Working Paper n. 201. Centre for Development Economics - CDE. Department of Economics. Delhi School of Economics. New Delhi, India, 2011.

MARZALL, K. e ALMEIDA, J. Indicadores de sustentabilidade para agroecossistemas: estado da arte, limites e potencialidades de uma nova ferramenta para avaliar o desenvolvimento sustentável. Cadernos de Ciência \& Tecnologia, v. 17, n. 1, p. 41-59, 2000.

MICCO, A. e PANIZZA, U. Bank ownership and lending behavior. Economics Letters, v. 93, n. 2, 2006.

MYRDAL, G. Beyond the Welfare State: economic planning and its international implications. New Haven, Connecticut: Yale University Press, 1960.

PACINI, C. et al. Evaluation of sustainability of organic, integrated and conventional farming systems: a farm 
and field-scale analysis. Agriculture, Ecosystems and Environment, v. 95, p. 273-288, 2003.

PAMPLONA, L. M. P. BNDES e o desenvolvimentismo do século 21: estado, democracia e sustentabilidade. Inc. Soc., v. 6, n. 1, p. 52-68, 2012.

PANNELL, D. J. e GLENN, N. A. A framework for the economic evaluation and selection of sustainability indicators in agriculture. Ecological Economics, v. 33, p. 135-149, 2000.

PARRA-LOPEZ, C. et al. A systemic comparative assessment of the multifunctional performance of alternative olive systems in Spain within an AHPextended framework. Ecological Economics, v. 64, n. 4, p. 820-834, 2008.

REZENDE. G. C. Crescimento econômico e oferta de alimentos no Brasil. Revista de Economia Política, v. 6, n. 1, 1986.

. Ajuste externo e agricultura no Brasil, 19811986. Revista Brasileira de Economia, v. 42, n. 2, p. 101137, 1988.

RIGBY, D. et al. Constructing a farm level indicator of sustainable agricultural practice. Ecological Economics, v. 39, p. 463-478, 2001.

SACHS, I. Desarrollo sustentable, bio-industrialización descentralizada y nuevas configuraciones ruralurbanas: los casos de India y Brasil. Pensamiento Iberoamericano, v. 46, p. 235-256, 1990.

. Caminhos para o desenvolvimento sustentável. Rio de Janeiro: Ed. Garamond, 2000.

SALOMÃO, L. A. Bancos públicos e desenvolvimento. Rio de Janeiro: IPEA, 2010.

SANT'ANNA, A. A. et al. Bancos públicos sustentam o crédito bancário no Brasil. Visão do Desenvolvimento, n. 63, BNDES, 2009.
SHLEIFER, A. State versus private ownership. Journal of Economic Perspectives, v. 12, p. 133-150, 1998.

SHLEIFER, A. e VISHNY R. Politicians and firms. Quarterly Journal of Economics, v. 109, p. 995-1025, 1994.

SIVAKUMAR, M. Gross Capital Formation and GDP growth in Indian Agriculture Sector. Munich Personal RePEc Archive-MPRA, Paper n. 46946, Tamilnadu, Índia, 2013.

WARNER, A. M. A Framework for Efficient Government. Working Paper. Research Department, International Monetary Fund - IMF, 2013.

WARNER, A. M. Public Investment as an Engine of Growth. Working Paper. Research Department, International Monetary Fund - IMF, Washington, 2014.

WEGELIN, M. J. C. O impacto da politica de crédito do BNDES no desenvolvimento econômico e social dos municípios brasileiros. Dissertação (Mestrado em Economia) - IBMEC. Rio de Janeiro, 2014.

WORLD BANK. Where is the Weath of Nations? Measuring Capital for the $21^{\text {st }}$ Century. Washington, 2006.

. The changing wealth of nations: measuring sustainable development in the new millenium. Washington, 2010.

. Relatório Anual do Banco Mundial. Washington, 2008.

World Commision on Environment and Development. Our Commun Future. Brundtland Report, 1987. Disponível em: <http://www.undocuments.net/wcedocf.htm >. Acesso em: fev. 2015.

YEYATI, E., MICCO, A. e PANIZZA, U. A reappraisal of state-owned banks. Economía, v. 7, n. 2, p. 209-247, 2007.

ZHEN, L. e ROUTRAY, J. K. Operational indicators for measuring agricultural sustainability in developing countries. Environmental Management, v. 32, n. 1, p. 34-46, 2003. 
A distinção entre $H_{0}, H_{1}$ e $H_{2}$ é feita via uso das seguintes estatísticas de teste propostas por Caner e Hansen (2001):

a) Uma estatística $t$ para $\rho_{1}, t_{1}$, utilizada para testar a hipótese nula de raiz unitária, $H_{0}: \rho_{1}=\rho_{2}=0$, contra a alternativa de estacionaridade apenas no regime 1 , isto é; $H_{2}: \rho_{1}<0$ e $\rho_{2}=0$.

b) Uma estatística $t$ para $\rho_{2}, t_{2}$, utilizada para testar a hipótese nula de raiz unitária, $H_{0}: \rho_{1}=\rho_{2}=0$, contra a alternativa de estacionaridade apenas no regime 2, isto é; $H_{2}: \rho_{1}=0$ e $\rho_{2}<0$.

c) Uma estatística de Wald unicaudal, $R_{1 T}=t_{1}^{2} I_{\left(\hat{\rho}_{1}<0\right)}+t_{2}^{2} I_{\left(\hat{\rho}_{2}<0\right)}$, utilizada para testar a hipótese nula de raiz unitária, $H_{0}: \rho_{1}=\rho_{2}=0$, contra a alternativa $H_{2}: \rho_{1}<0$ e $\rho_{2}<0$.

Os valores críticos para as estatísticas $R_{1 T}, t_{1}$ e $t_{2}$ estão tabulados em Caner e Hansen (2001). Foram tabulados valores críticos assintóticos e, para melhorar a inferência em amostras pequenas, valores críticos por "bootstrap".

É importante mencionar que simulações de monte carlo realizadas por Caner e Hansen (2001) mostram que, na presença de raiz unitária parcial, os testes baseados nas estatísticas $t$ têm muito mais potência (e melhor tamanho) que o tradicional teste ADF e que o teste baseado na estatística $R_{1 T}$. Na presença de estacionaridade pura (estacionaridade nos dois regimes), os testes $t$ ainda possuem mais potência que o teste ADF quando existem efeitos de "threshold" nos outros parâmetros do modelo (1). Isto levou Caner e Hansen a concluir que os testes $t$ são capazes de discriminar corretamente os casos de raiz unitária pura, raiz unitária parcial e estacionaridade pura. 
